\title{
Construcción racional y autónoma de valores y habilidades sociales
}

\section{Silvia López}

\section{$\mathcal{D} \mathcal{M a}$}

La enseñanza de las habilidades sociales requiere el uso de diversas técnicas y procedimiento. En este artículo se relacionan las más directamente implicadas en un curriculum de educación moral orientado bacia el desarrollo de la autonomía. Se propone una actividad para el primer ciclo de Educación Primaria. Además, se ofrecen prototipos de actividades para otros tramos educativos.

\section{INTRODUCCION}

El aprendizaje de las habilidades sociales se ha estudiado desde el ámbito de las teorías neoconductistas, principalmente en el marco del aprendizaje social (Bandura y Walters 1964, 1974; Bandura 1977), que consideran el desarrollo moral como el aprendizaje de la conducta socialmente aceptada, haciendo incidencia en la adquisición e internalización de las normas y valores vigentes en los diferentes ámbitos de relación. Estas teorías afirman que las habilidades sociales pueden ser aprendidas como cualquier otra conducta, mediante un proceso de obervación, identificación, imitación y reproducción de conductas.

Como complemento a las teorías anteriores, hemos de considerar las posturas de carácter más cognitivo (Piaget, Kolhberg...) si queremos que la educación moral no se reduzca a la transmisión unilateral de conductas, actitudes y valores, sino que tenga como finalidad la construcción racional y autónoma de los valores. Así, el desarrollo de la competencia social de niños/as y adolescentes tiene una importancia primordial tanto a nivel cognitivo como desde el punto de vista de la formación moral.

\section{LAS HABILIDADES SOCIALES}

Las diferentes aportaciones teóricas citadas nos permiten hablar de las habilidades sociales como el conjunto de comportamientos interpersonales que va aprendiendo la persona y que configuran su competencia social en los diferentes ámbitos de relación. Las habilidades sociales permiten a la persona encontrarse más aceptada y valorada por los otros ya que manifiesta conductas de 
carácter asertivo, es decir, conductas que expresan sus sentimientos, deseos, opiniones... de una forma abierta y sincera, respetando a la vez todas estas manifestaciones en los otros. Todo este conjunto de conductas que va aprendiendo la persona le permitirán, tomar decisiones teniendo en cuenta los propios intereses y los de los otros; elaborar juicios críticos compartiendo criterios y opiniones; resolver los problemas personales y de relación con actitudes de comprensión y colaboración; y establecer relaciones satisfactorias para uno mismo y los demás.

Un curriculum de educación ético-moral debe potenciar el aprendizaje de las habilidades sociales, y en especial el desarrollo de estos comportamientos y actitudes que posibilitan una interacción social positiva en los diferentes ámbitos de relación (escuela, barrio, familia...). Las habilidades sociales que debería contemplar un curriculum de educación moral pueden agruparse en:

- Habilidades prosociales y sociales, necesarias para la interrelación y comunicación recíproca y positiva con los demás: mantener una conversación, formular preguntas, pedir favores...

- Habilidades afectivas, que posibilitan el conocimiento de los sentimientos personales, así como también las emociones y sentimientos de los demás.

- Habilidades para solucionar conflictos, es decir, habilidades alternativas a la agresión y que permiten hacer frente al estrés, posibilitando la resolución de problemas personales, interpersonales y sociales.

- Habilidades para conjugar autonomía y aceptación de obligaciones.

- Habilidades personales de autorregulación y autocontrol de la conducta que facilitan una actuación de acuerdo con los razonamientos y juicios morales desarrollados.

\section{PROCESO DE ENSEÑANZA APRENDIZAJE DE LAS HABILIDADES SOCIALES. ASPECTOS PRÁCTICOS}

La enseñanza de las habilidades sociales requiere la utilización de diversas técnicas y procedimientos. Esto supone un proceso que se inicia ofreciendo información a propósito de la habilidad que se quiere trabajar; a continuación se ofrecen ejemplos - modelos - positivos y/o negativos de la habilidad; estas dos primeras fases se complementan con representaciones de situaciones donde se practiquen los comportamientos observados; estas representaciones han de ir seguidas de una información que incluye el refuerzo social. Por último, para favorecer la generalización de las conductas aprendidas en la clase, se han de proponer ejercicios que permitan su transferencia y generalización a otros ámbitos de relación.

En todo este proceso el educador/a ha de potenciar situaciones donde se pongan en juego determinadas capacidades y destrezas de carácter prosocial. Nos estamos refiriendo a situaciones que posibiliten la adopción de perspectivas sociales y la empatía; los procesos de imitación de modelos positivos y la utilización del razonamiento, ya que estas capacidades contribuyen a la internalización de las habilidades sociales que se trabajan.

A continuación se presenta - a propósito de una actividad concreta- el proceso que puede seguir el/la educador/a para trabajar las habilidades sociales en el aula. El ejemplo que proponemos está pensado para el ciclo inicial de la Primaria (6-8 años) y corresponde a las habilidades sociales necesarias para una adecuada interrelación con el medio físico, actividad que se incluiría en el ámbito temático «Ciencia, técnica y ecología» 


\section{ACTIVIDAD: VAMOS DE EXCURSION}

\section{Primera y segunda fase: Información y Modelaje}

En estas edades consideramos que la primera y segunda fase se han de dar conjuntamente, estableciendo una relación directa entre la información a propósito de la habilidad que queremos trabajar y los ejemplos de la misma. De esta forma, creemos que se favorece la atención de los niños/as, así como la interiorización de los modelos que se presentan.

En la primera fase el maestro/a ofrecerá información sobre la habilidad social, indicando en qué consiste, es decir, los comportamientos que ha de manifestar el alumno/a. También explicará las ventajas al manifestar determinados comportamientos y los inconvenientes al no manifestarlos o manifestar otros que se oponen.

En la segunda fase el educador/a proporcionará ejemplos directos de la habilidad social que se trabaje.

En estas edades (6-8 años), la información y los modelos pueden presentarse mediante un cuento que vaya acompañado de dibujos que ejemplifiquen tanto los comportamientos negativos como los positivos, de forma que los niños/as puedan compararlos. Con esto lo que se pretende es trabajar el aprendizaje por imitación de los modelos positivos, los cuales se valoran respecto a los otros.

Cuento: Vamos de excursión (modelo negativo)

La clase de Javier fueron de excursión al campo. Cuando llegaron les gustó mucho.

Hacia un día fantástico, el río estaba muy limpio, había mariposas, hormigas, caracoles, ardillas, pájaros... Se lo pasaron estupendo Jugaron, pasearon, comieron,...
Pero a la hora de irse, todo era diferente. Algunos niños y niñas habían tirado papeles al suelo, latas al río...

A ellos no les importaba, porque ya se iban y no iban a volver.

\section{Orientaciones para la presentación del modelo negativo.}

Se reparte una ficha con los dibujos y el cuento, que puede ser leído por el profesor/a por los mismos alumnos.

El maestro/a hará reflexionar sobre las consecuencias del comportamiento de los protagonistas del cuento, mediante preguntas como:

- ¿Cómo ha quedado el bosque?

- ¿Está igual que cuando llegaron los niños y niñas?

- ¿Qué pensarán los animales que viven en el campo?

- ¿Cuando volvamos nos gustaría encontrarnoslo así? ¿Por qué?

\section{Cuento: Vamos de excursión (modelo positivo)}

La clase de Montse ha ido de excursión al campo. Cuando llegaron les gustó mucho.

Hacia un día fantástico, el río estaba muy limpio, había mariposas, hormigas, caracoles, ardillas, pájaros... Se lo pasaron muy bien. Jugaron, pasearon, comieron...
Tuvieron mucho cuidado. Los papeles los metieron en unas bolsas, igual hicieron con las latas.

Cuando se fueron todo estaba tan limpio y bonito como cuando llegaron. 
Orientaciones para la presentación del modelo positivo

Se ha de seguir el mismo proceso que al trabajar el modelo negativo, haciendo incidencia en las conductas positivas que manifiestan los protagonistas.

\section{Orientaciones acerca de los dos modelos}

Una vez se han presentado los dos modelos, es importante realizar una serie de preguntas y comentarios con el fin de que los niños y niñas reflexionen sobre las consecuencias de los comportamientos que se presentan en las dos historias. Algunas de las preguntas podrían ser:

- ¿Cuál de las dos historias te gusta más? ¿Por qué?

- ¿Por qué los niños y niñas de la clase de Javier tiran las cosas al suelo y los de la clase de Montse en una bolsa?

- ¿Qué pasaría si todas las clases hicieran lo mismo que la clase de Javier?

- ¿Has visto niños y niñas que hagan lo mismo?

Con la última pregunta se invita a los alumnos a explicar experiencias vividas que ejemplifiquen los comportamientos observados en los modelos. A partir de aquí el educador/a deberá hacer una síntesis de las ventajas de mantener limpio el entorno y de los inconvenientes de no respetarlo, proponiendo algunas normas que se pueden seguir para cuidar y conservar el entorno más próximo. Sería interesante que estas normas o guías conductuales se representen en dibujos y se expongan en la clase para que los alumnos/as puedan observar y recordar lo que se debe hacer.

\section{Tercera fase. Representación}

Después de que los alumnos/as hayan observado los comportamientos adecuados, se trata de que puedan ensayarlos mediante pequeñas representaciones. El educador/a o bien los niños/as, dependiendo de la edad, proponen nuevas situaciones a representar perfilando el carácter de los personajes, de forma que queden bien claros los comportamientos que han de manifestar.

Ejemplo de situación a representar:

Hoy es el aniversario de Todos los niños y niñas Ana. $\mathrm{Ha}$ traido unas bolsitas con caramelos para repartir a sus compañeros antes de salir·al patio. de su clase están muy contentos y han salido corriendo al patio.
¿Qué han hecho los niños y niñas con los papeles de los caramelos?

¿Cómo ha quedado el patio?

\section{Cuarta fase: Retroalimentación y Refuerzo}

Consiste en ofrecer al alumno/a información sobre como ha representado el papel y como ha manifestado los comportamientos y conductas que se están trabajando (refuerzo social).

A partir del ejemplo que se ha representado, se pueden hacer preguntas relacionadas con su comportamiento cuando van al patio o en diferentes contextos, donde es necesario respetar y mantener limpio el entorno.

\section{Quinta fase: Generalización y transferencia}

Esta fase consiste en aplicar, fuera del contexto de aprendizaje, las habilidades sociales adquiridas. Con el ejemplo que estamos trabajando se podría organizar alguna salida al campo, al zoo..., y previamente recordar lo que es necesario hacer para mantener limpio el entorno. En este sentido conviene tener las nor- 
mas o guias conductuales expuestas en la clase. A partir de aquí se puede proponer realizar algún ejercicio de autorregulación y autocontrol de la conducta a propósito del tema trabajado.

\section{Otras actividades}

«La radio del abuelo»

Ambito temático: Relaciones en el ámbito familiar. Curso: $4 .^{\circ}$ de E.G.B.

El abuelo de Paco ha vivido muchos años solo y está acostumbrado a poner la televisión y la radio con el volumen muy alto. A Paco le molesta oír la televisión cuando estudia o juega en su habitación. Esta tarde estaba haciendo los deberes con Sonia, una niña de su clase, y ha salido de la habitación para decirle su abuelo que apagara la radio. Mira lo que han dicho:

Paco: En esta casa nunca se puede estudiar por culpa de tu radio.

Abuelo: Te pasas el día gritando con tu hermana y ahora dices que te molesta mi radio.

Paco: Ya puedes apagarla inmediatamente porque no hay manera de hacer los deberes.

Abuelo: Bueno, hombre. Ahora la bajo.

Paco: No quiero que la bajes porque la seguiré oyendo. Quiero que la apagues.

El abuelo de Paco ha vuelto a su habitación, ha bajado un poco el volumen y ha continuado escuchando la radio.

¿Qué te parece el diálogo de Paco con su abuelo? ¿Crees que Paco lo ha hecho bien? ¿Por qué?

¿Qué quería conseguir Paco?

¿Qué ha hecho para conseguirlo?

¿Qué crees que ha pensado el abuelo de su nieto?

¿Por qué crees que el abuelo pone el volumen de la radio tan alto?

Y Paco, ¿por qué le debe haber hablado de esa manera a su abuelo?

¿A tí alguna vez te ha pasado algo parecido?

Explica como fue:

¿Crees que Paco hubiera podido hablar con su abuelo de otra manera?

A continuación poneos por parejas y imaginad un diálogo entre Paco y su abuelo, en el que ninguno de los dos se enfade con el otro y en el que Paco consiga lo que quiere: no oir la radio mientras estudia.

Ahora podéis memorizar el diálogo que habéis inventado y representarlo ante vuestros compañeros.

\section{Orientaciones generales para la elaboración de nuevos ejercicios.}

Es interesante que las actividades de habilidades sociales se realicen posteriormente a otras actividades que den información o hagan reflexionar sobre el tema que se está tratando. Hablamos de actividades como por ejemplo, comprensión crítica, clarificación de valores, dilemas morales... que introducen y sitúan al alumno en la temática a propósito de la cual se realizará un trabajo de habilidades sociales, quedando estas así contextualizadas. Seguir las siguientes pautas puede ayudar al educador/a en la elaboración de ejercicios de habili. dades sociales: 
1) Instrucciones/información: explicación de la habilidad; ventajas e inconvenientes de poseerla

2) Modelaje/aprendizaje por imitación; Ejemplos de manfiestación de la habilidad (modelo) en diferentes ámbitos de relación (familia, escuela, iguales...). Normas o guías conductuales.

3) Representación de papeles: ejemplos de situaciones que el alumno/a representará para poner en práctica la habilidad observada.

4) Retroalimentación/refuerzo: preguntas y comentarios que se pueden hacer después de la representación

5) Generalización/transferencia: propuesta de «deberes» para poner en práctica la habilidad trabajada fuera del contexto.

\section{Referencias}

Álvarez, A.; AlvarezMonteserin, M.a A.; Cañas, A.; Jiménez, S.; Petit, M. ${ }^{\text {a }}$ (1990). Desarrollo de las habilidades sociales en niños de 3.6 años. Aprendizaje Visor.

CARrLlo, I «Habilidades sociales» en MARTINEZ, M. \& PUIG, J. M. ${ }^{\text {a }}$ (coord) (1991). La educación moral. Perspectivas de futuro y técnicas de trabajo Ed. Graó. ICE Universitat de Barcelona. pp. 131-142.

Goldstein, A. P.; SprafKInG, R.; Gershayw, N. J.; KLeIN, P. (1989). Habilidades sociales y autocontrol en la adolescencia Martínez Roca.

Michelson, L.; Sugal, D. P.; Wood, R. P.; Kazdin, A. L. (1987) Las babilidades sociales en la infancia. Martínez Roca.

\section{Construcción racional y autónoma de valores y habilidades sociales \\ Silvia López \\ CL\&E, 1992, 15, pp. 93-98}

Resumen: El desarrollo de la competencia social de niños/as y adolescentes tiene una importancia primordial tanto a nivel cognitivo como desde el punto de vista de la formación moral. Se puede hablar de las habilidades sociales como el conjunto de comportamientos interpersonales que va aprendiendo la persona y que configuran su competencia social en los diferentes ámbitos de relación. Las habilidades sociales, permiten a la persona encontrarse más aceptada y valorada por los otros ya que manifiesta conductas de carácter asertivo, es decir, conductas que expresan sus sentimientos, deseos, opiniones..., de una forma abierta y sincera. Un curriculum de educación ético-moral debe potenciar el aprendizaje de las habilidades sociales, y en especial el desarrollo de estos comportamientos que posibilitan una interacción social positiva en los diferentes ámbitos de relación (escuela, ba. rrio, familia...).

Datos sobre el autor: Silvia López, es pedagoga y actualmente goza de una beca de Formación de Personal Investigador del Ministerio de Educación y Ciencia. También es miembro del Grupo de Investigación en Educación Moral (G.R.E.M.).

Dirección: Departamento de Teoría e Historia de la Educación, Facultad de Pedagogía, Universidad de Barcelona, Baldiri Reixac, s/n 08028 Barcelona. Tel (93) 3333466.

(C) De todos los artículos deberá solicitarse por escrito autorización de CL\&E y de los autores para el uso en forma facsímil, fotocopia o cualquier otro medio de reproducción impresa. CL\&E se reserva el derecho de interponer las acciones legales necesarias en aquellos casos en que se contravenga la ley de derechos de autor. 


\section{El diagnóstico de situación, una técnica para el análisis de alternativas y la valoración de sus consecuencias}

\section{Jesús Vilar}

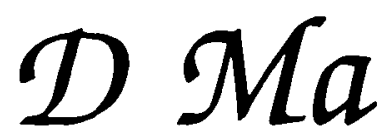

Se consideran las etapas fundamentales para el diagnóstico de situación y los pasos a seguir en su aplicación. Esta técnica que intenta ayudar a los alumnos a pensar, analizar, criticar y juzgar la realidad que les rodea se ejemplifica en actividades dirigidas en especial a los alumnos del segundo ciclo de la Educación Secundaria, ofreciéndose, además, prototipos de actividades para otros tramos educativos.

\section{INTRODUCCION}

El diagnóstico de situación es una técnica de educación moral que tiene por objeto el desarrollo de la capacidad de valorar las diferentes alternativas que se presentan en una situación problemática así como las posibles consecuencias de cada una de ellas, a partir de una situación de diálogo organizado.

En este sentido, el diagnóstico de situación contiene características de tres tipos de procedimientos habitualmente utilizados en educación moral:

- Aquellos que tiene por objeto desarrollar en el sujeto su capacidad de análisis. Conviene distinguir en primer lugar los hechos que se presentan en una situación y las diversas opciones que se barajan, así como los valores que se implican en cada una de ellas.

- Aquellos que tienen por objeto desarrollar el juicio moral. Derivados de la incertidumbre sobre la validez y bondad de la decisión tomada una vez que se analizan las consecuencias de cada decisión y porque el diálogo organizado es una fuente de novedad cuando las opiniones que se presentan son diversas o controvertidas.

- Aquellos que tienen por objeto el desarrollo de la capacidad de llegar a acuerdos mediante el diálogo y la argumentación. El diagnóstico o valoración final requiere que los diferentes miembros del grupo realizen la actividad de 
diagnóstico mediante el uso de la argumentación y la exposición de razones,

las valoren críticamente y lleguen a algún tipo de síntesis.

El punto de partida de esta técnica es, como en los dilemas morales, una situación real o hipotética donde un protagonista ha tomado una decisión sobre una situación problemática de difícil solución, en la que entran en conflicto diversos valores más o menos básicos.

La diferencia fundamental con los dilemas morales es que la técnica de diagnóstico guarda más relación con los contenidos de los valores que se implican en la situación o que se presentan en la materia que se está tratando que con la forma de juicio que se está emitiendo. Es decir, mientras los dilemas se dirigen específicamente a discutir las razones que han movido al protagonista a tomar una decisión determinada, el diagnóstico de situación busca sobre todo que se analice la decisión en sí, que se consideren los valores que se ponen en juego en esa decisión, que se busquen otras alternativas posibles a la decisión que se ha tomado y que se valoren las consecuencias que se derivarían de tomar alguna de ellas.

Por otra parte, esta técnica resulta muy útil para desarrollar la capacidad de análisis de situaciones complejas en las que se deben tener en cuenta un númeo más o menos importante de factores que influyen en esa situación, así como sus posibles consecuencias. Fomenta la habilidad para discernir entre alternativas diversas al considerar las consecuencias que se derivan de cada una de ellas así como los valores que se ponen en juego.-

Esta técnica sigue básicamente tres etapas fundamentales:

- En primer lugar, se presenta una situación conflictiva a los alumnos y se les pide que en grupo o de manera individual busquen criterios para juzgar la situación. En relación con los criterios establecidos, deben pensar otras alternativas posibles que se habrían podido tomar en esa situación.

- En segundo lugar, se valoran las consecuencias de las diferentes alternativas posibles que los alumnos han presentado, en relación con los valores que se ponen en juego en cada una de ellas.

- En tercer lugar, se procede a emitir un juicio para esas situaciones -que será el diagnóstico propiamente dicho,- después de haber discutido colectivamente las repercusiones o consecuencias de la decisión tomada inicialmente o de las decisiones posibles.

Estas tres etapas fundamentales se pueden desglosar en seis pasos:

1. Identificar y clarificar los valores implicados en la situación que se discute. Conviene centrar el problema de manera que queden claros los valores que se ponen en discusión y los argumentos que se aportan.

2. Buscar hechos y datos significativos para el problema que se debate que puedan servir de alternativa a la decisión inicial. Es importante distinguir entre hechos y valores.

3. Comprobar el grado de confianza que nos merecen las posibles alternativas después de considerar los valores que cada una de ellas ponen en juego.

4. Clarificar la relevancia de las alternativas que se han presentado y si son útiles para ayudar a clarificar el problema que se está tratando.

5. Alcanzar una postura consensuada entre todos los miembros del grupo que analizan el tema, a la luz de lo aportado en las etapas anteriores.

6. Evaluar la decisión que se tomó inicialmente viendo si es aceptable en sí misma y en sus consecuencias previsibles. 
En síntesis, podemos decir que esta técnica intenta ayudar a los alumnos a pensar, analizar, criticar y juzgar la realidad que les rodea. También les ayuda a buscar y formar criterios y a emitir juicios estudiando los diferentes niveles en los que se mueve la persona, así como los diversos ángulos de los que se puede enfocar un mismo acontecimiento.

\section{EJEMPLO DE ACTIVIDAD}

La actividad que se pone de ejemplo está seleccionada para personas de 14-16 años aproximadamente y trata el tema de la dificultad que tienen los jóvenes para decidir su futuro cuando se plantean posibilidades muy distantes entre ellas y que afectan la cuestión laboral.

Iremos mostrando como se desarrolla la actividad en relación con los pasos que anteriormente hemos anotado.

\section{Presentación del problema}

Se reparte un texto donde está explicada la situación problemática que se debe diagnosticar. Este texto ha de mostrar claramente cuales son las alternativas que se presentan así como el núcleo del problema. La narración ha de acabar mostrando la decisión que toma el protagonista de la historia que se expone.

También se debe tener preparada una lista de preguntas orientativas para realizar el diagnóstico.

Título de la actividad: «El futuro, el trabajo y el estudio»

«La actividad que os proponemos tiene por objeto que reflexionéis sobre las dudas que se os presentan a la hora de decidir vuestro futuro.» ¿Qué haré? ¿Estudiaré, me dedicaré a trabajar, podré compaginar las dos actividades?»

Para trabajar este tema os proponemos que analicéis esta situación de la que más tarde deberéis hacer un diagnóstico o una valoración. Seguramente, este problema ya se os ha presentado o sabéis de algún amigo o amiga vuestro que se encuentra ante estas dudas.

«Rosa es una chica que estudia $3 .^{\circ}$ de BUP. Nunca ha sido una estudiante excepcional, pero siempre ha trabajado con mucha voluntad y por eso ha obtenido muy buenos resultados en aquellas materias que le gustaban más.

Desde pequeña ha estado muy interesada por el estudio de la naturaleza y hace tiempo que decidió que estudiaría Biológicas en la universidad.

A pesar de su interés por el tema, se ha dado cuenta que cada vez le cuesta más interesarse por el resto de materias de BUP. No se siente motivada y además tiene poco tiempo para estudiar. Esto le está produciendo muchas duchas sobre como plantearse el futuro, sobre todo cuando ve que todavía le queda acabar el curso que está haciendo y todo COU. Además, no toda la biología le interesa por igual y ve que deberán pasar aún algunos años antes de que se pueda dedicar específicamente a aquello que le interesa realmente.

Paralelamente, hace unos meses ha comenzado a trabajar en una oficina durante las tardes. Este trabajo dificulta su concentración y le permite disponer de menos tiempo libre. Además, el otro día le propusieron trabajar todo el día a partir del momento que ella crea conveniente. Es un trabajo monótono que le interesa relativamente, pero es en una empresa sólida y seria, por lo cual puede contar con trabajo fijo y con posibilidades de mejorar su posición laboral. 
Ella es consciente que cada vez está menos interesada por los estudios de bachillerato (aunque le interese la biología) y se está planteando dejar de estudiar para ponerse a trabajar. Esta posibilidad le permitirá tener dinero, tiempo libre y liberarse del nerviosismo y el malestar que le produce la situación en que se encuentra respecto de los estudios. Quizás algún día retomará los estudios. Además, tampoco está claro que en la actualidad una persona pueda trabajar de aquello que ha estudiado.

De todos modos, tiene bastantes dudas. Teme que la seguridad de un trabajo fijo la aleje de lo que realmente le interesa y desea más claramente: estudiar algunos aspectos muy concretos de la naturaleza.

Después de pensarlo mucho, Rosa ha decidido acabar el curso y ponerse a trabajar.»

\section{Como organizarse}

Un aspecto importante es la manera como se organizan los alumnos para garantizar que se realize un trabajo mínimamente correcto y eficaz. No hay que olvidar que la capacidad de trabajo y la motivación del grupo, que es lo que determina en última instancia que se puedan obtener buenos resultados de la actividad, está directamente relacionada con una planificación adecuada de la tarea que se pretende realizar.

En este sentido, conviene tener en cuenta algunos aspectos. En primer lugar la organización del grupo. Conviene hacer grupos pequeños que esten compensados (que todos los miembros del grupo puedan hacer aportaciones y que estas aportaciones generen nuevas dudas entre ellos) y se invita a los alumnos que intenten responder a las preguntas que orientan la realización del diagnóstico.

«Haced grupos de cinco personas. Cada grupo discutirá las preguntas que se anotan a continuación antes de elaborar el diagnóstico definitivo.»

\section{Clasificación de las preguntas orientadoras}

Relacionado con el apartado anterior, otro de los factores importantes a tener en cuenta es que los alumnos no se sientan desconcertados ante las preguntas que deben ir respondiendo y no decrezca el nivel de atención respecto de la tarea.

Así pues, en primer lugar mostraremos algunas preguntas que pueden utilizar la persona que dirige la sesión para orientar la reflexión de los alumnos en el trabajo de diagnóstico. Esas preguntas deben ser consideradas como facilitadoras de la reflexión de los alumnos y serán utilizadas cuando decrezca el nivel de discusión, ya sea porque no se comprende lo que se pide en alguno de los pasos del diagnóstico, ya sea porque hay algún paso que les resulta más difícil de resolver.

Preguntas orientadoras para valorar las alternativas y pensar las consecuencias:

1. ¿Crees que vale la pena estudiar materias que no te interesan durante mucho tiempo, sabiendo que lo que más te interesa tardará mucho en llegar?

2. ¿Crees que vale la pena dedicar demasiados esfuerzos a estudiar si no tienes demasiadas garantías de trabajar en el campo que has estudiado?

3. ¿Crees que los estudios escolares y de BUP. ofrecen lo que los alumnos necesitan?

4. ¿Qué crees que deberían ofrecer los estudios de BUP? 
5. ¿Qué crees que les sobra?

6. ¿Crees que en vuestra edad tenéis el soporte necesario por parte de los adultos con los que tenéis relación? Explica en qué crees que sí y en qué crees que no.

7. ¿Crees que la cuestión económica es decisiva para decidir si continuar estudiando o no? Piensa si no lo es nunca, si depende de las circunstancias o si lo es siempre.

\section{Organización de las preguntas dirigidas a los alumnos}

Cuando se presenta el texto a los alumnos donde se muestra la situación, debemos aportar también el listado de preguntas o actividades que se deben seguir para hacer el diagnóstico. Estas cuestiones deben ir siguiendo, en la medida de lo posible, los pasos que anteriormente anotábamos sobre como realizar el diagnóstico.

Así pues, a continuación mostraremos las preguntas orientadoras que se hacen a los alumnos y las iremos relacionando con los pasos que se deben seguir en la realización del diagnóstico.

Preguntas que se plantean a los alumnos y relación de las mismas con los pasos del diagnóstico:

1. Identificar y clarificar los valores implicados en la situación que se discute.

- Si tu fueras Rosa, ¿qué harías?

- Piensa razones a favor y razones en contra sobre la decisión tomada por Rosa.

- Considerad las consecuencias de esta decisión.

- Pensad qué ha valorado Rosa para tomar esta decisión.

2. Buscar bechos y datos significativos para el problema que se debate que puedan servir de alternativa a la decisión inicial.

- Buscad el núcleo del problema. Realmente, ¿cuál es el problema, cuáles són las variables que lo condicionan y cuáles son los valores que se ponen de manifiesto?

- Una vez habeis encontrado el núcleo del problema y las variables que lo condicionan, pensad alternativas posibles a la decisión que ha tomado Rosa.

- Analizad los valores que se ponen en juego en cada una de las alternativas que habéis pensado, es decir, mostrad qué es lo que se valora con mayor insistencia en cada una de ellas (por ejemplo: paciencia, capacidad de autocontrol y de fijarse objetivos a largo plazo, beneficio económico, estatus social, sinceridad con uno mismo, tranquilidad personal...)

3. Comprobar el grado de confianza que nos merecen las posibles alternativas después de considerar los valores que cada una de ellas ponen en juego.

- Valorad el grado de cada alternativa en función de los valores que se han seguido para proponer las alternativas.

4. Clarificar la relevancia de las alternativas que se han presentado y si son útiles para ayudar a clarificar el problema que se está tratanto.

- En función de las consecuencias que habéis analizado, ordenad las posibles alternativas de la más satisfactoria a la menos satisfactoria. Poned en cada una de ellas lo que se valora con más importancia.

5. Alcanzar una postura consensuada entre todos los miembros del grupo que analizan el tema, a la luz de lo aportado en las etapas anteriores. 


\section{4}

- A partir del trabajo que habeis realizado hasta este momento, haced una propuesta consesuada sobre qué decisión creéis que habría sido más correcta y por qué.

6. Evaluar la decisión que se tomó inicialmente viendo si es aceptable en sí misma $y$ en sus consecuencias previsibles.

- Elaborad un diagnóstico final sobre la decisión que ha tomado Rosa. Justificad el diagnóstico.

\section{Elaboración de una conclusión final}

En el apartado B hemos anotado la necesidad de organizar la clase en pequeños grupos y esta organización la hemos mantenido durante toda la actividad. Para finalizar la actividad, conviene que cada uno de los grupos manifieste las conclusiones a que han llegado y que elaboren conjuntamente, ahora ya todos los alumnos de la clase juntos, una conclusión final donde se muestre el diagnóstico colectivo que se realiza y las razones que lo justifican.

Conviene remarcar que, en general, este tipo de actividades acaban siempre con una conclusión colectiva sobre el trabajo que se ha realizado. El esfuerzo final de llegar a un acuerdo colectivo puede significar que todo el trabajo anterior cobre sentido y sea provechoso. Si queda interrumpido en algún punto, puede ocurrir facilmente que los esfuerzos realizados tanto individualmente como en los pequeños grupos se diluya y se pierda por la razón de no haberse materializado en una conclusión que haga de resumen respecto del tema concreto que se discute como de las habilidades puestas en funcionamiento para la realización del ejercicio.

«Ahora que ya habéis elaborado el diagnóstico, haced una puesta en común de las conclusiones a que cada grupo ha llegado. A continuación haced un mural donde se exponga una conclusión conjunta de toda la clase. Si no hay un acuerdo más o menos amplio, conviene que en el mural se incluyan las opiniones más destacables que hayan aparecido en la discusión final.»

\section{ALGUNOS EJEMPLOS DE DIAGNOSTICO DE SITUACION}

\section{«Juzguemos los casos»}

El Sr. Sumoi es propietario de una empresa immobiliaria. Hace dos años terminó un bloque de 60 viviendas y dos parkings. Los negocios le han ido muy bien pero, esperando que la evolución del mercado de la vivienda fuera todavía positiva, dejó por vender 10 pisos y un parking de 70 plazas. Ahora, al cabo de dos años, los ha puesto a la venta con un incremento de 1,5 millones por cada plaza de parking y tres millones por cada piso.

1. ¿Consideras correcta la actuación de este personaje? ¿Por qué?

2. ¿Qué valores crees que ha considerado para tomar esta decisión?

3. ¿Qué piensas que debería haber hecho? Anota alguna alternativa y los valores que se tienen en cuenta en cada una de ellas.

4. ¿A quién puede repercutir la actuación del Sr. Sumoi? Piensa en las consecuencias de las alternativas que has anotado.

5. ¿Piensas que este personaje ha actuado con profesionalidad? ¿Por qué? 
6. ¿Cuál crees que habría sido la actuación más correcta? ¿Por qué?

7. Haz una valoración final del caso.

\section{«La corrección de exámenes»}

Aurora es profesora de Ciencias Sociales en una escuela. Este fin de semana tiene que corregir todos los exámenes finales de sus alumnos para poder tener entregadas las notas el lunes pero precisamente le han llamado unos amigos, que hace mucho tiempo que no ve, para que vaya con su marido a pasar el fin de semana a la torre que aquellos tienen en Port de la Selva. Ella tiene muchísimas ganas de verlos y de salir, así que le pide a su hija, que está estudiando segundo de historia, que le corrija los exámenes y le ponga las notas.

1. ¿Consideras correcta la actuación de este personaje? ¿Por qué?

2. ¿Qué valores crees que ha considerado a la hora de tomar esta decisión?

3. ¿Qué piensas que debería haber hecho? Apunta algunas alternativas y los valores que se tienen en cuenta en cada una de ellas.

4. ¿A quién puede repercutir la decisión que ha tomado Aurora? ¿En qué sentido? Piensa las consecuencias de las alternativa que has anotado.

5. ¿Piensas que este personaje ha actuado con profesionalidad? ¿Por qué?

6. ¿Cuál crees que habría sido la actuación más correcta? ¿Por qué?

7. Haz una valoración final del caso.

\section{Referencias}

Martinez, M.; Puig, J.M. (1989). Educación y democracia. Laertes. Barcelona.

AA.VV. (1990). Etica i escola. El tractament pedagógic de la diferéncia. Rosa Sensat. Barcelona. 